\title{
La modernisation de l'enseignement de la chimie dans le réseau de l'Université du Québec : réflexion sur un projet de collaboration
}

\author{
Denis Bussières ${ }^{1}$, Université du Québec à Chicoutimi \\ Daniel Maltais ${ }^{2}$, École nationale d'administration publique
}

\section{Introduction}

Une expérience de collaboration entre huit professeurs ouvrant dans cinq constituantes universitaires du réseau de l'Université du Québec (UQ) : l'UQAC (Chicoutimi), l'UQAM (Montréal), l'UQAR (Rimouski), l'UQTR (Trois-Rivières) et la TÉLUQ (Télé-université) a été réalisée à l'initiative du professeur Guy Collin ${ }^{3}$ de l'UQAC. Elle a eu lieu de mai 1999 à avril 2001 et visait à moderniser l'enseignement de la chimie dans les programmes de premier cycle dans le réseau de l'Université du Québec (UQ), lequel se heurtait essentiellement à deux problèmes majeurs. La première problématique est la difficulté intrinsèque de l'apprentissage de la chimie, la deuxième étant le manque de ressources humaines dédiées à l'enseignement de la chimie dans la plupart des constituantes de l'UQ. Plus précisément, on notait:

- le peu d'admissions aux programmes en région et un nombre important d'abandons et d'insuccès, en particulier à l'UQAM;

- l'érosion marquée du corps professoral en chimie, discipline pourtant essentielle au support et au développement de plusieurs autres champs d'études dont l'environnement, les pâtes et papiers, les ressources renouvelables, l'océanographie, etc.;

- l'isolement relatif des départements de chimie à l'intérieur même de leur université;

- l'émergence et l'explosion du développement des technologies de l'information et des communications (TIC), donc de nouveaux outils disponibles pour l'enseignement, favorisant la collaboration entre des ressources professorales géographiquement dispersées.

\section{Rassemblement des acteurs et objectifs}

L'initiateur du projet commença par recruter les participants pour ensuite les réunir, une fois l'avantprojet accepté par l'administration du réseau de l'UQ. Cette première rencontre a permis à chacun de faire connaissance et de découvrir les intérêts des autres participants. À partir de ces constats, il fut proposé de développer le projet FODAR-Chimie qui :

- faciliterait l'apprentissage et l'appropriation des concepts théoriques en chimie par les étudiants;

- permettrait à des professeurs dispersés dans le réseau de l'UQ de collaborer à une œuvre collective et pédagogique;

- rendrait accessible un plus grand nombre de professeurs en chimie du réseau de l'UQ à l'ensemble des étudiants de chimie;

- favoriserait l'appropriation des TIC par les professeurs et les étudiants de chimie, ces technologies offrant des possibilités considérables en ce qui concerne la compréhension et la diffusion des connaissances aux étudiants;

- mettrait à la portée des étudiants toute la richesse d'information que recèle l'Internet sur les connaissances en chimie.

Compte tenu de la diversité des expertises, il a été convenu que le groupe concentrerait ses efforts sur le développement de deux cours de trois crédits (deux 
volets). Les huit professeurs ont été associés à l'un ou l'autre des deux volets suivants du projet :

A. la conception et la réalisation d'outils d'apprentissage du cours cinétique chimique (un professeur de l'UQAC, un professeur de l'UQTR et un professeur de l'UQAR);

B. la conception et la réalisation d'outils d'apprentissage de la chimie organique (deux professeurs de l'UQAM, un professeur de l'UQTR et un professeur de l'UQAR).

Le professeur de la TÉLUQ a agi comme conseiller en matière de multimédia pédagogique et a été impliqué plus particulièrement dans le projet sur la cinétique chimique.

\section{L'initiateur du projet commença par recruter les participants pour ensuite les réunir, une fois l'avant-projet accepté par l'administration du réseau de I'UQ.}

Mentionnons qu'au départ, la plupart des personnes associées au projet utilisaient déjà largement les TIC dans leur pratique d'enseignement. Ainsi, chacune d'elles avait déjà développé ses propres outils de façon parallèle et indépendante. En fait, le projet FODAR-Chimie venait appuyer et encourager la production d'outils, mais surtout leur mise en commun. Du coup, le projet permettait d'assurer la pérennité et le développement des programmes de chimie offerts dans les constituantes concernées, en particulier en région où les clientèles sont peu nombreuses. L'UQAM y voyait pour sa part un moyen de retenir davantage d'étudiants dans ses programmes.

\section{La réalisation du projet}

$\mathrm{Au}$ début du projet, les professeurs ont dû partager de l'information tant sur leurs compétences respectives que sur leur intérêt à travailler et à utiliser les TIC. Cela donna rapidement lieu à des échanges entre les professeurs rattachés à l'intérieur de chacun des deux volets du projet et facilita le partage d'idées et les apports constructifs de chacun.
Il faut comprendre que le partage d'informations et d'outils pédagogiques n'est ni évident ni fréquent chez les professeurs. Malgré une matière commune, les outils pédagogiques que développe un professeur portent en quelque sorte son "empreinte », laquelle s'appuie sur son bagage spécifique d'expertise et d'expérience, ses propres champs d'intérêt et ses propres préoccupations, ce qui explique en partie que les outils pédagogiques soient rarement partagés et difficilement « interchangeables ». Il n'est donc pas étonnant de trouver de nombreux ouvrages portant sur une même matière, en l'occurrence la chimie, qui la présentent de manières différentes, et de constater qu'un professeur choisira l'ouvrage de référence pour ses étudiants en fonction de sa propre approche.

\section{Au début du projet, les professeurs ont dû partager de l'information tant sur leurs compétences respectives que sur leur intérêt à travailler et à utiliser les TIC.}

Cette période d'échanges initiale a inauguré le démarrage des deux volets mentionnés plus tôt. Chacun des volets devait être réalisé par une petite équipe de professeurs volontaires qui s'est rapidement mise à la tâche. Même si, fondamentalement, l'objectif premier des deux équipes était le même, à savoir la modernisation de l'enseignement de la chimie, l'approche adoptée par chacune d'elles a été différente, au moins au cours de la première année. À l'UQAM, l'objectif spécifique était de mettre à la disposition des étudiants du matériel en ligne, de créer des exercices, des applications qui permettaient aux étudiants, fort occupés par ailleurs, de pouvoir accéder en tout temps à des outils de formation pédagogique complémentaires. À l'UQAC, la démarche a été différente puisqu'elle visait d'abord à développer des outils utilisables par le professeur pour améliorer et moderniser son enseignement. Ce qui est intéressant, c'est qu'au cours de la seconde année du projet, chaque équipe s'inspira de l'approche de l'autre. Ainsi, lors de la seconde année, l'UQAM développa des outils pédagogiques pour le professeur en format PowerPoint, alors que l'UQAC réalisa des exercices avec corrigés et des outils interactifs pour les étudiants. On peut donc affirmer que l'émulation des 
deux équipes s'est vraiment réalisée durant cette deuxième année. Finalement, bien qu'inattendue, une troisième réalisation a vu le jour, soit le développement d'un outil multimédia permettant de visualiser les mouvements internes d'une molécule. $\mathrm{Si}$, en apparence, cette réalisation s'éloignait quelque peu des objectifs de départ, elle allait contribuer au développement d'autres cours, notamment à l'UQAC.

Il est utile de noter que les participants ne se sont rencontrés qu'à quelques reprises pour la réalisation de tout le projet. Ce n'est qu'à la fin, soit après les deux ans qu'a duré le projet, qu'une réunion générale de tous les participants a eu lieu. Donc, tout au long du travail, le courriel et le téléphone ont été les principaux outils de communication.

\section{L'outil de modélisation de mouvements moléculaires qui a été réalisé a servi de prétexte pour le développement de matériel pour le cours de chimie théorique que l'on a aussi déposé sur un site Web.}

Chacune des deux équipes a été complètement libre d'adopter sa propre démarche de fonctionnement interne, et chaque participant avait à organiser son travail localement, en fonction de la nature de ses responsabilités et de son environnement organisationnel immédiat. Pour ce qui est du volet $\mathrm{A}$, trois professeurs ont participé à sa réalisation en se partageant le travail: ainsi, alors que le travail du professeur de l'UQAC s'est davantage concentré sur les outils multimédias en support à l'enseignant, celui du professeur de la TELUQ a porté essentiellement sur le développement complémentaire d'un outil multimédia animé. Quant au volet B, les deux professeurs de l'UQAM ont davantage travaillé ensemble en se partageant les tâches axées sur le support aux étudiants et en mettant régulièrement en commun les portions accomplies.

\section{Les résultats obtenus}

Dans le cadre du volet A, un cours complet de trois crédits a été adapté sous un format électronique; les notes de cours incluant tableaux, graphiques et équations ont été élaborées et rendues disponibles sur un site $\mathrm{Web}^{4}$. Ce site comprend donc les notes de cours avec problèmes résolus pour 12 chapitres, ce qui correspond à environ 200 pages (papier). De plus, ces documents ont servi de base à la production de présentations de format PowerPoint ${ }^{T M}$ correspondant à chaque chapitre. S'inspirant des travaux de l'autre équipe, l'équipe rattachée au volet $\mathrm{A}$ a également développé et mis sur le site Web des exercices et des quiz avec solutions. L'outil de modélisation de mouvements moléculaires qui a été réalisé a servi de prétexte pour le développement de matériel pour le cours de chimie théorique que l'on a aussi déposé sur un site Web ${ }^{5}$. Un des professeurs a même poursuivi le travail entrepris en adaptant ses notes pour les cours de thermochimie ${ }^{6}$ et d'introduction à la physique atomique et nucléaire ${ }^{7}$.

Pour ce qui est du volet $B$, l'équipe a développé une série d'exercices et de problèmes avec les solutionnaires pour chacun des 13 chapitres du cours de base en chimie organique, le tout disponible à partir d'une plateforme $W e b C T^{T M^{8}}$. La deuxième année, plus de 600 diapositives sous format PowerPoint ${ }^{T M}$ ont été réalisées pour appuyer l'enseignement des professeurs du cours de chimie organique ${ }^{9}$. Tout ce matériel a également été rendu disponible sur support CD-ROM pour permettre une plus grande flexibilité en classe et en faciliter la diffusion auprès des étudiants. Encouragés et très stimulés par ces résultats, le même travail a été entrepris pour le second cours de chimie organique.

\section{Les participants considèrent que l'apport des TIC, très présentes dans le développement des outils pédagogiques réalisé dans le cadre du projet, leur a permis de progresser, comme professeurs.}

\section{L'analyse de l'expérience du point de vue des professeurs qui y ont participé ${ }^{10}$}

Les participants considèrent que l'apport des TIC, très présentes dans le développement des outils pédagogiques réalisé dans le cadre du projet, leur a permis de progresser, comme professeurs, en permettant notamment d'aller plus rapidement et plus 
loin dans la matière, de raffiner les explications et d'améliorer la démonstration et la compréhension de certaines parties plus complexes. Certains ont même souligné la valeur promotionnelle des outils développés auprès des étudiants et des étudiantes du niveau collégial, une clientèle potentielle du programme de chimie offert par leur université.

Il ressort en outre des discussions avec les professeurs participants que trois éléments principaux ont contribué plus directement à l'atteinte des objectifs et au succès du projet. Premièrement, les qualités et les compétences de la personne en charge du projet apparaissent comme un ingrédient de base. On fait référence à son leadership, à son dynamisme communicatif, au respect et à la confiance mutuelle qu'elle a su promouvoir. La clarté des résultats attendus des participants fut le deuxième élément mentionné : les objectifs du projet étaient très explicites et chacun connaissait précisément ce qu'on attendait de lui. Enfin, le troisième élément concerne un mode de fonctionnement qui laissait toute la souplesse et la marge de manœuvre nécessaires à la réalisation des activités prévues.

Par ailleurs, les professeurs participants ont signalé que certains aspects du projet auraient pu être renforcés. Ainsi, plusieurs auraient voulu une meilleure diffusion des résultats, en particulier auprès de collègues non participants. Ce faisant, on aurait pu mieux les sensibiliser au projet et à ses objectifs, susciter leur intérêt et, dans certains cas, contrer le scepticisme ou le peu d'ouverture que certains collègues ont pu manifester. Dans le même ordre d'idées, on aurait trouvé intéressant que le projet suscite davantage d'échanges entre les participants et qu'il donne lieu à l'organisation d'un colloque ou d'un forum pour favoriser les échanges entre professeurs de chimie, susciter des maillages, etc.

On a aussi signalé qu'il aurait été utile de prévoir une forme d'évaluation ou de validation externe des outils développés, surtout s'ils sont destinés à une utilisation plus étendue. Dans ce cas, une certaine homogénéité aurait été requise pour s'assurer que les outils soient utilisables par des personnes différentes. Des participants ont souligné que plusieurs d'entre eux devaient prendre ou étaient sur le point de prendre leur retraite. Dans de telles circonstances, il aurait été préférable de constituer une équipe présentant un meilleur équilibre intergénérationnel, à savoir des professeurs en début de carrière, à mi-carrière et en fin de carrière.

Enfin, plusieurs ont fait remarquer que le développement d'outils pédagogiques exige un investissement très important en temps. Or, les universités de moins grande taille ne disposent souvent pas de la marge de manœuvre nécessaire pour dégager une ressource aux fins d'un projet de cette envergure, alors qu'elles éprouvent tout autant, sinon davantage, le besoin de développer de tels outils. En d'autres mots, les conditions de participation à un tel projet, notamment au chapitre des moyens que les petites universités peuvent mettre à contribution, sont souvent fragiles et difficiles à remplir. Du coup, on convient que ce genre de projet, en rapprochant des constituantes, est une avenue intéressante, car il permet justement de constituer une masse critique de ressources dont ne dispose pas une petite université.

\section{Une analyse du projet du point de vue des modes de collaboration}

Parmi les discours à la mode en gestion, il y a celui de la nécessité d'une plus grande collaboration entre des organisations qui ont tendance à travailler « en silo ». Ce discours met l'accent sur l'horizontalité, la concertation et le partenariat en laissant entendre que l'avenir appartiendra aux organisations capables de travailler "en réseau ». Mais en premier lieu, nous devons préciser les différents types ou degrés possibles de collaboration. Pour ce faire, le tableau 1 résume brièvement les points de vue de plusieurs auteurs. L'ordre de présentation de ces modes n'est pas fortuit, la concertation et le partenariat constituant les deux modes de collaboration les plus sophistiqués et les plus exigeants pour les organisations au regard des dimensions retenues. Comme le lecteur peut se rendre compte, la classification repose sur l'évaluation subjective de quelques paramètres contextuels. Cette évaluation peut très bien être différente d'un observateur à un autre pour une même situation de collaboration. Nonobstant cette particularité, cette grille nous permet d'utiliser un cadre commun de discussion et de s'assurer que les termes sont bien définis. 


\section{Tableau 1 - Quatre modes de collaboration entre organisations *}

\begin{tabular}{|c|c|c|c|c|}
\hline & \begin{tabular}{|c|}
$\begin{array}{c}\text { Transfert et échange } \\
\text { d'informations }\end{array}$ \\
\end{tabular} & Consultation & Concertation & Partenariat \\
\hline Pourquoi? & $\begin{array}{l}\text { Faire connaître les } \\
\text { services ou produits }\end{array}$ & $\begin{array}{l}\text { Obtenir une validation } \\
\text { ou enrichir un } \\
\text { processus de réflexion }\end{array}$ & $\begin{array}{l}\text { Assurer la complé- } \\
\text { mentarité des } \\
\text { missions, l'harmoni- } \\
\text { sation des stratégies }\end{array}$ & $\begin{array}{l}\text { Offrir de meilleurs } \\
\text { services; profiter } \\
\text { d'une occasion }\end{array}$ \\
\hline $\begin{array}{l}\text { Pouvoirs et } \\
\text { responsabilités }\end{array}$ & Inchangés & Inchangés & À respecter & À partager \\
\hline Convergence d'intérêts & Minimale & À explorer & À expérimenter & $\begin{array}{l}\text { À déployer } \\
\text { pleinement }\end{array}$ \\
\hline Confiance & Minimale & À développer & À renforcer & À consolider \\
\hline Enjeux et coûts & Faibles & & & Élevés \\
\hline Résultats anticipés & $\begin{array}{l}\text { Une meilleure } \\
\text { information }\end{array}$ & $\begin{array}{l}\text { Une meilleure } \\
\text { compréhension; } \\
\text { une réduction de } \\
\text { l'incertitude }\end{array}$ & $\begin{array}{l}\text { Moins ou pas de } \\
\text { chevauchements; de } \\
\text { meilleurs ajustements } \\
\text { mutuels }\end{array}$ & $\begin{array}{l}\text { Une production } \\
\text { impossible ou } \\
\text { problématique sans } \\
\text { l'apport de l'autre }\end{array}$ \\
\hline Processus en cause & Communication & $\begin{array}{l}\text { Compréhension/ } \\
\text { Apprentissage }\end{array}$ & Collaboration étroite & $\begin{array}{l}\text { Mise en commun de } \\
\text { ressources }\end{array}$ \\
\hline
\end{tabular}

* Ce tableau et le schéma 1 présenté plus loin s'inspirent de Georges Burdeau (1981) ${ }^{11}$, des notes de Lionel Ouellet et de Marc-André Dionne, ex-professeurs à l'ENAP, et de celles de Daniel Maltais, professeur à l'ENAP, ainsi que d'un avis du Conseil supérieur de l'éducation ${ }^{12}$ sur le partenariat.

En fait, l'importance de la collaboration entre certaines organisations n'est pas nouvelle. Ce qui est nouveau, ce sont certains éléments de contexte qui rendent la collaboration plus souhaitable. Ainsi, beaucoup d'organisations n'ont plus les moyens de leurs ambitions et envisagent plus facilement de partager leurs ressources pour réaliser leurs projets. Dans d'autres cas, la nature des problèmes rencontrés fait en sorte qu'il est impossible d'envisager de les résoudre à moins de travailler avec des "partenaires ». Les problèmes qu'essayait de résoudre le projet FODAR-Chimie illustrent bien qu'il y avait dans ce cas précis "matière à collaboration", sinon entre organisations, du moins entre professeurs appartenant à des organisations différentes mais soucieux de développer leur expertise en enseignement de la chimie.

En se référant au contenu du tableau 1 et à celui du schéma 1, et en se rappelant le déroulement du projet
FODAR-Chimie, il apparaît assez clairement que celui-ci correspond essentiellement au deuxième mode de collaboration axé sur la " consultation ». Nous avons en effet signalé plus tôt dans ce texte que les participants venaient d'institutions différentes, que leur rôle respectif fut convenu et que la charge de travail fut distribuée à l'intérieur d'équipes. Nous sommes donc amenés à penser que chaque petite équipe chargée d'un volet fonctionnait, conformément aux distinctions proposées quant aux modes de collaboration, plutôt en mode " concertation ». Les membres des deux équipes avaient des intérêts convergents et s'assuraient de chevauchements minimums en harmonisant autant que possible les tâches à effectuer pour l'atteinte de leurs objectifs respectifs. Durant le déroulement du projet, les pouvoirs et responsabilités de chaque membre d'une équipe étaient respectés et la confiance a pu se développer et se renforcer. 


\section{Schéma 1 - Représentation des différents modes de collaboration et de certains paramètres}

QUATRE MODES DE COLLABORATION ENTRE ORGANISATIONS : DE

L'ÉCHANGE D'INFORMATION AU PARTENARIAT (CADRE CONCEPTUEL)

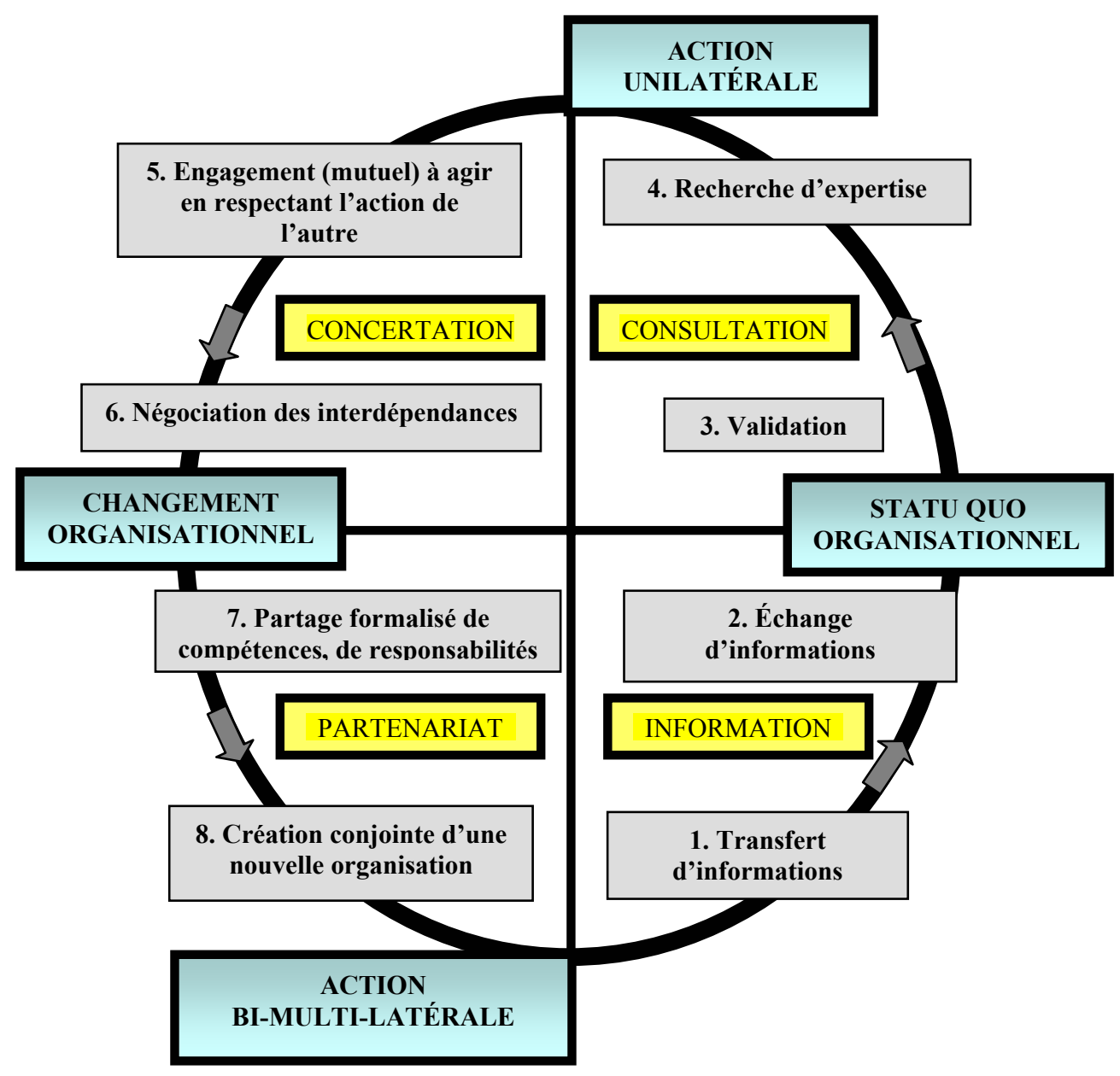

De plus, chaque équipe s'est inspirée des travaux et réalisations de l'autre équipe pour complémenter son travail. Ceci a permis de bonifier les résultats obtenus tout en respectant les compétences, pouvoirs et responsabilités de chacun. Une telle concertation a été possible à l'intérieur des équipes mais, du point de vue de l'ensemble du projet, les travaux et résultats sont tout de même restés très distincts. Il s'agit de se rappeler les remarques des participants sur la non-uniformité des outils développés, les différences de structure et de présentation, le manque de validation du matériel. Pour l'ensemble du projet, nous considérons plutôt que la collaboration est restée au niveau de la consultation entre les participants des deux équipes. Cela a certes contribué au développement d'une connaissance et d'une confiance mutuelles, ce qui permet d'espérer que des collaborations plus exigeantes, comme une pleine concertation ou un partenariat, se développeront dans l'avenir.

\section{Conclusion}

Tout en étant plus fréquentes depuis quelques années, les collaborations entre professeurs œuvrant dans des institutions d'enseignement différentes sont bien en deçà de ce qu'elles pourraient être. Nous avons voulu montrer qu'il était possible de les encourager, notamment dans le domaine de l'ensei- 
gnement, en comprenant mieux ce qui peut expliquer le succès d'un projet de collaboration récent entre professeurs provenant de trois universités différentes. Le projet FODAR-Chimie a été réalisé et a atteint ses objectifs avec, en prime, quelques résultats positifs imprévus. Le leadership, le dynamisme et la confiance suscités par le coordonnateur du projet ainsi que la clarté des objectifs visés ont été des ingrédients essentiels à sa bonne réalisation.

\section{Durant le déroulement du projet, les pouvoirs et responsabilités de chaque membre d'une équipe étaient respectés et la confiance a pu se développer et se renforcer.}

En référence aux quatre modes de collaboration brièvement présentés dans cet article, il apparaît que le mode qui a caractérisé le fonctionnement des équipes fut celui de la consultation, un mode de collaboration qui a respecté le degré d'autonomie requis des personnes impliquées tout en permettant l'atteinte des résultats recherchés. Qui plus est, ce mode de collaboration a permis à ces personnes de mieux se connaître et d'envisager de collaborer plus étroitement dans des projets subséquents de concertation ou de partenariat. Cette possibilité est d'ailleurs une réalité puisqu'un projet de même nature a été accepté pour 2001-2003. De nouvelles ressources professorales se sont greffées, démontrant du coup la volonté de poursuivre dans le même sens.

Plusieurs projets actuellement en chantier verront leur aboutissement prochain, contribuant ainsi à renouveler la dynamique d'enseignement de la chimie et à rapprocher les constituantes concernées. C'est là un acquis de première importance qui devrait consolider et positionner favorablement le programme de chimie parmi les disciplines offertes dans le réseau de l'UQ.

\section{Notes et références}

1 Denis Bussières est professeur à l'Université du Québec à Chicoutimi. Il est détenteur d'un Ph.D. en chimie de l'Université of Western Ontario (1985), d'un DESS en administration publique de l'ÉNAP (2003). Ses intérêts couvrent, outre la chimie inorganique et environnementale, l'enseignement et les méthodes pédagogiques utilisées en chimie.

2 Daniel Maltais est professeur à l'École nationale d'administration publique. Il possède un baccalauréat en sciences politiques de l'Université de Montréal (1976), un MBA de l'École des HEC (1983) et un doctorat en administration publique de l'Université Southern California (1996). Ses intérêts couvrent les nouvelles formes d'organisation gouvernementales, les modes de collaboration entre les organisations gouvernementales et les organisations privées (avec ou sans but lucratif) et l'administration publique (en particulier sous l'angle de la performance organisationnelle). Ses recherches actuelles portent sur les cabinets ministériels et sur les compétences de gestion des cadres supérieurs gouvernementaux.

3 Collin, G. (1999). Modernisation de l'enseignement de la chimie, formulaire de présentation des propositions au FODAR, mars.

4 www.uqac.ca/chimie/cinetique_chimique/

5 www.uqac.ca/chimie/Chimie_théorique/

6 www.uqac.ca/chimie/Thermochimie/

7 www.uqac.ca/chimie/Physique_atom/

8 www.er.uqam.ca/nobel/dep_chim/chi1301/outils/

9 www.er.uqam.ca/nobel/dep_chim/Diapos-CHI1301/Diaporama-CHI1301.ppt

10 Nous remercions M. Serge Bonneau qui a consulté les professeurs associés au projet. Cette partie de l'article résume les propos rapportés.

11 Burdeau, G. (1981). Traité de science politique, tome III : La dynamique politique, vol. II : La dialectique de l'ordre et du mouvement, chap III : De la lutte à la gestion, Paris, LGDJ.

12 Conseil supérieur de l'éducation (2002). Les universités à l'heure du partenariat, avis au ministre de l'Éducation, mai, $114 \mathrm{p}$. 